\title{
More Accurate Size Contrast Judgments in the Ebbinghaus Illusion by a Remote Culture
}

\author{
Jan de Fockert and Jules Davidoff \\ Goldsmiths College, University of London
}

\author{
Joel Fagot and Carole Parron \\ Université de la Méditerranée
}

\author{
Julie Goldstein \\ Goldsmiths College, University of London
}

\begin{abstract}
The Ebbinghaus (Titchener) illusion was examined in a remote culture (Himba) with no words for geometric shapes. The illusion was experienced less strongly by Himba compared with English participants, leading to more accurate size contrast judgments in the Himba. The study included two conditions of inducing stimuli. The illusion was weaker when the inducing stimuli were dissimilar (diamonds) to the target (circle) compared with when they were similar (circles). However, the illusion was weakened to the same extent in both cultures. It is argued that the more accurate size judgments of the Himba derive from their tendency to prioritize the analysis of local details in visual processing of multiple objects, and not from their impoverished naming.
\end{abstract}

Keywords: Ebbinghaus illusion, local precedence, size contrast judgments, cross-cultural

Cross-cultural studies have repeatedly suggested differences in the way two-dimensional images are coded (for reviews, see Deregowski, 1989; Nisbett \& Miyamoto, 2005). Particularly interesting are the finding that people from different cultures do not seem to access the global structure of pictorial objects equally readily and the finding that some cultures prioritize the analysis of the local details of an object (Deregowski, 1989), whereas others prioritize the analysis of the global features (Nisbett \& Miyamoto, 2005).

The current research explored cross-cultural differences in the Ebbinghaus (Titchener) illusion, a perceptual phenomenon where the size of a central target object is affected by the size of surrounding inducers (see Figure 1). The illusion is sensitive to manipulations of the figural similarity (Choplin \& Medin, 1999; Coren \& Miller, 1974) and conceptual similarity (Coren \& Enns, 1993) between the target and the inducers. Less illusion is normally found with increasing dissimilarity between the target and the inducers, suggesting the target and the inducers were grouped together less strongly. Population differences (e.g., with autistic children) have been observed for the illusion within Western society (Dakin \& Frith, 2005; Happé, 1996; Happé, Briskman, \& Frith, 2001; although see Ropar \& Mitchell,

Jan de Fockert, Jules Davidoff, and Julie Goldstein, Department of Psychology, Goldsmiths College, University of London, London, England; Joel Fagot and Carole Parron, Institut de Neurosciences Cognitives de la Méditerranée, Centre National de la Recherche Scientifique, Université de la Mediterranée, Marseille, France.

We thank Alan Pickering for statistical advice. This research was funded by a major research fellowship from the Leverhulme Trust (F/07 605/D), the European Union project Stages in the Evolution and Development of Sign Use (012-984), and Eurocores Origins of Man, Language and Languages.

Correspondence concerning this article should be addressed to Jan de Fockert, Department of Psychology, Goldsmiths College, University of London, New Cross, London SE14 6NW, United Kingdom. E-mail: j.de-fockert@gold.ac.uk
1999), and given the known cultural differences in context sensitivity (Nisbett \& Miyamoto, 2005), a cross-cultural population may show a similar attenuated illusion if they have difficulty in accessing global structures of displays.

We conducted a comparison of the Ebbinghaus illusion between English and Himba participants. The Himba, who speak a dialect of Herero, are a seminomadic people in a remote area of northern Namibia, who have extremely limited access to Western technology and no formal education. They are cattle herders, and like those from other similar African cultures (Davidoff, 1975, pp. 88-89; Eckl, 2000; Evans-Pritchard, 1940, p. 48), precise recognition of individual cattle from their markings is critical to Himba culture. Nisbett, Peng, Choi, and Norenzayan (2001) argued that such practices can influence perceptual style. They contended that Westerners pay more attention to detail (analytical processing) than do Asian populations, who are more sensitive to the context (holistic processing). However, it could be that the Himba pay even greater attention to detail than Westerners because of their need to know the identity of individual cattle. In this respect, we note that the process of individuating animals requires processes that are clearly different from the global processing that is used in individuating faces (McNeil \& Warrington, 1993). Thus, our first hypothesis concerned a potential tendency among the Himba to prioritize the analysis of local details. Specifically, we predicted that if there is a local processing preference in the Himba, this attention to the target would lead to a reduction in the illusory effect caused by the inducers and would therefore lead to a reduction in the magnitude of the Ebbinghaus illusion. Furthermore, if the reduction derives from a change in attention, it could well be independent of effects due to inducer similarity.

There is another aspect of the Himba language that might predict a different pattern of performance in shape processing. The Himba possess very few shape terms (Roberson, Davidoff, \& Shapiro, 2002) and no words for geometric shapes. In a replication 

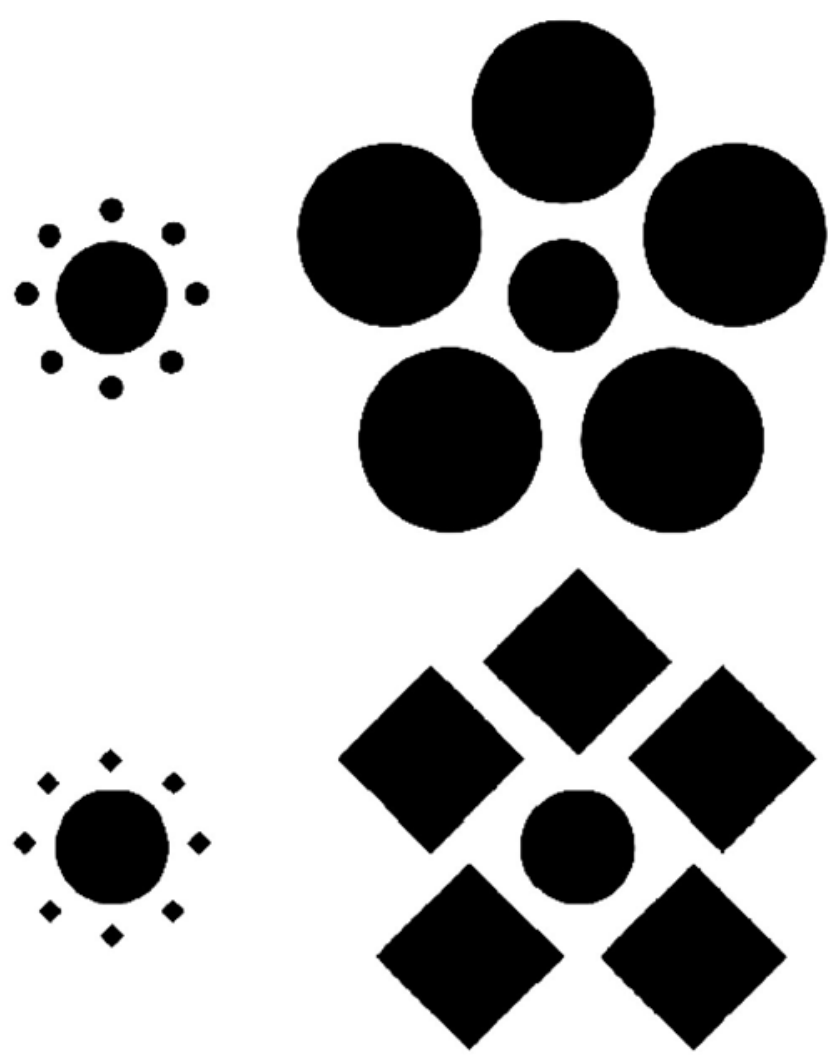

Figure 1. Illustration of the Ebbinghaus illusion. In this example, the four central target circles are equal in size, yet a target surrounded by smaller inducers (left of display) appears to be larger than a target surrounded by larger inducers (right of display), implying that the size contrast illusion depends on perceptual binding. The illusion is stronger for inducers that are similar (top of display) compared with those that are dissimilar (bottom of display) to the target.

of the category learning study by Rosch (1973), Roberson et al. (2002) found that the Himba, unlike Westerners, learned irregular square, circle, and triangle shapes to be part of a category as easily as they learned the prototype examples. Furthermore, in a shapesorting task with the same materials, they grouped together stimuli that had strong local similarities, even if they were from different Western shape categories (square, circle, or triangle). These findings suggest that the absence of clear linguistic distinctions among different geometric shapes has perceptual implications, so that two shapes with strong local similarities, yet belonging to different Western categories, can be grouped together by the Himba.

Our second hypothesis therefore concerned a linguistic effect on grouping by similarity. It is derived from the larger question of whether concepts are relative to language, a theory for which there is increasing evidence from the study of perception (for reviews, see Roberson et al., 2002; Roberson, Davies, \& Davidoff, 2000). The effect of similarity between the target and the inducers (Coren $\&$ Miller, 1974) makes the Ebbinghaus illusion particularly suitable to test the effects of differences in linguistic labeling on perception. On the basis of linguistic relativity, it would be predicted that the Himba participants should experience more illusion than the English participants when the target and the inducers are different geometric shapes (e.g., circle vs. diamond). The Himba might even see an equal extent of illusion regardless of the similarity between the target and the inducers.

\section{Method}

\section{Participants}

Participants were 8 ( 3 men and 5 women) adult monolingual Himba from an isolated region in Northern Namibia (mean estimated age $=30$ years 10 months, range $=20-45$ years). Their language contains no words for geometric shapes, like circles and squares. Further participants were 8 ( 3 men and 5 women) native English speakers (mean age $=31$ years 3 months, range $=23-54$ years). The English participants were Goldsmiths College staff volunteers or paid students. The Himba were rewarded in kind. No cases of abnormal vision were reported.

\section{Stimuli}

Stimulus configurations were presented in black on white cardboard paper $(20 \times 15 \mathrm{~cm})$ and consisted of a central target circle surrounded by inducing stimuli, which were large or small circles (similar inducers) or large or small diamonds (dissimilar inducers; see Figure 1). The center-to-center distance between the target and each inducer was $32 \mathrm{~mm}$ for the large inducers and $16 \mathrm{~mm}$ for the small inducers. Two stimulus configurations were presented simultaneously to participants on each cardboard. In the small inducers configuration, circle or diamond inducers were presented with a diameter of $4 \mathrm{~mm}$, and the size of the central target circle remained constant at $19 \mathrm{~mm}$ in diameter. In the large inducers configuration, circle or diamond inducers were presented with a diameter of $32 \mathrm{~mm}$, and the target diameter ranged from $17.66 \mathrm{~mm}$ to $22.15 \mathrm{~mm}$, with $0.66-\mathrm{mm}$ steps. Thus, there were two stimulus cards (17.66 and $18.32 \mathrm{~mm}$ ) in which the target circle in the large inducers condition was smaller than the target circle in the small inducers condition (target size differences of -1.32 and $-0.66 \mathrm{~mm}$, respectively), one stimulus card in which the two targets were equal in size (target size difference of $0 \mathrm{~mm}$ ), and five stimulus cards in which the target circle in the large inducers condition was the larger one (target size differences of $0.66,1.32$, $1.98,2.64$, and $3.30 \mathrm{~mm}$ ). Thus, relative to the target in the small inducers condition of $19 \mathrm{~mm}$, the size of the target circle in the large inducers condition was $93.1 \%, 96.5 \%, 100.0 \%, 103.5 \%, 106.9 \%$, $110.4 \%, 113.9 \%$, and $117.4 \%$, respectively. The asymmetry in the stimulus set makes use of the fact that a reverse illusion does not occur (large inducers never produce the illusion of a larger target). Thus, some conditions in a symmetrical array would be highly redundant. The asymmetrical stimulus set also has the advantage that the middle stimulus is not veridical. Thus, neither random performance nor any strategy based on the range of target sizes in the large inducers condition leads to veridical performance.

\section{Procedure}

Blocks of eight practice trials were administered in which participants saw two circles: one that varied in diameter from 17.66 to 22.15 $\mathrm{mm}$ and another that remained constant at $19 \mathrm{~mm}$. Participants were instructed to point at the larger circle, and training continued until they reached $75 \%$ accuracy within a block. All participants needed only one block of practice trials to reach criterion, except for one Himba participant who required two. After training, participants were pre- 


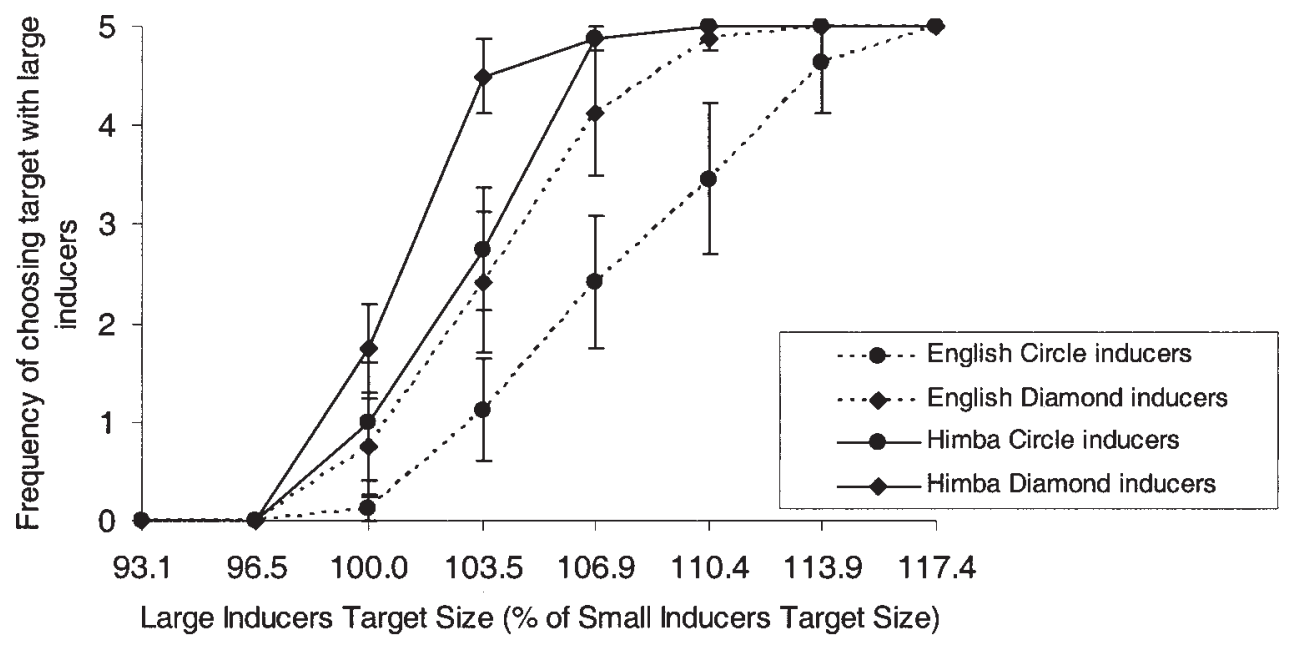

Figure 2. Mean frequency of choosing the target with large inducers, as a function of culture, inducer shape, and large inducers target size. Veridical equality was at large inducers target size $100 \%$. Error bars represent standard error of the mean.

sented with a block of 40 test trials for each inducing shape condition (circles or diamonds, order counterbalanced across participants), with each block consisting of five randomly distributed trials of each target size in the large inducers configuration (ranging in size from 17.66 to $22.15 \mathrm{~mm}$ ) presented together with a small inducers configuration. Stimulus cards were presented in horizontal orientation, and both within each block and within each target size in the large inducers configuration, the small inducers target was equally likely to occur on the left or on the right of the display. On each presentation of the two stimulus configurations, participants were asked to point at the target circle they thought was larger. These instructions were given with the help of a naive interpreter for the Himba participants. Viewing distance was approximately $45 \mathrm{~cm}$. The experimenter immediately noted the answer down after every trial. During the test, participants received no feedback on the accuracy of their responses.

\section{Results}

For each participant, the frequency of choosing the target with large inducers was entered into a 2 (culture: English, Himba) $\times 2$ (inducer shape: circles, diamonds) $\times 8$ (large inducers target size: $93.1 \%, 96.5 \%, 100.0 \%, 103.5 \%, 106.9 \%, 110.4 \%, 113.9 \%$, $117.4 \%$ ) mixed analysis of variance, with repeated measures on the last two variables and participants as the random variable. Frequencies ranged from 0 (target with large inducers chosen on none of the five repetitions of a trial type) to 5 (target with large inducers chosen on all five repetitions of a trial type), so lower scores indicate more illusion. Not surprisingly, there was a main effect for large inducers target size, $F(3,38)=173.3, p<.001$ (Greenhouse-Geisser corrected); $\eta_{\mathrm{p}}{ }^{2}=.925$ : The target with large inducers was chosen more frequently when it was larger. There was also a main effect for inducer shape, $F(1,14)=22.75, p<$ $.01 ; \eta_{\mathrm{p}}{ }^{2}=.619$, with the diamond inducers producing less illusion than the circle inducers (see Figure 2). More important for the current investigation was a significant main effect for culture, $F(1$, 14) $=9.88, p<.01 ; \eta_{\mathrm{p}}{ }^{2}=.414$, with less illusion for the Himba participants (mean frequency of choosing the target with large inducers $=3.11$ ), compared with the English participants (mean frequency of choosing the target with large inducers $=2.43$ ). The main effect of culture is even more compelling because there was no Culture $\times$ Inducer Shape interaction, $F(1,14)=3.08, p>.1$, suggesting that the two cultures were similarly sensitive to variation in inducer shape. There were significant two-way Inducer Shape $\times$ Large Inducers Target Size interactions, $F(3,44)=4.46$, $p<.01$ (Greenhouse-Geisser corrected); $\eta_{\mathrm{p}}{ }^{2}=.241$, trivially because of ceiling and floor effects at the extreme large inducers target sizes (see Figure 2). For the same reason, there was a Culture $\times$ Large Inducers Target Size interaction, $F(3,38)=5.13$, $p<.01$ (Greenhouse-Geisser corrected); $\eta_{\mathrm{p}}{ }^{2}=.268$. The threeway interaction was not significant, $F(3,44)=2.24, p>.09$.

We also computed the point of subjective equality (PSE) for each participant. We fitted the data, using the inverse cumulative distribution for a standard normal distribution to estimate each participant's threshold for deciding that the target with large inducers was the larger one. ${ }^{1}$ There was a greater mean PSE for the English compared with the Himba participants, $t(14)=3.62, p<$ .01. On average, the $19-\mathrm{mm}$ target with small inducers was seen as the same size as a 20.061-mm target with large inducers by the English participants and was seen as the same size as a $19.296-\mathrm{mm}$

\footnotetext{
${ }^{1}$ We fitted the following model to each participant's data from the circle and shape inducer conditions:

$$
p=\varphi([k-d] / \sigma),
$$

where $p$ is probability of choosing the target with large inducers, $\varphi(z)$ is the inverse cumulative distribution function for a standard normal distribution, $k$ is the required threshold for deciding that the target with large inducers is the larger one ( $k=0$ means no illusion, positive $k$ values indicate illusion), $d$ is the difference between the radius of the two circles (in millimeters), and $\sigma$ is the standard deviation of the normally distributed noise, combined for the perceptual process (associated with the perceived difference in size between the two targets) and the decision process (normally distributed variability in the placement of the decision criterion).
} 
target with large inducers by the Himba participants. An analysis of the differences of PSEs from veridicality $(0-\mathrm{mm}$ size difference between the two target circles, large inducers target size 100\%) revealed that both cultures experienced the illusion in the circle inducer configuration-for the English, $t(7)=6.13, p<.001$; for the Himba, $t(7)=4.04, p<.01$ - but that only the English participants experienced the illusion in the diamond inducer configuration, $t(7)=3.71, p<.01$. The Himba participants' PSEs were not different from the veridical, $t(7)=1.93, p>.09$, in the diamond inducer configuration.

To directly test that overall size judgment accuracy was indeed greater in the Himba participants than in the English participants, we looked at how often the veridically larger target was selected by each participant, irrespective of whether this target had small or large inducers, and we calculated the overall accuracy score for each participant. Trials in which the size of the target with large inducers was identical to that of the target with small inducers (19 $\mathrm{mm}$ ) were excluded from this analysis, because there was no correct answer here. As predicted, size judgments were significantly more accurate among the Himba participants (mean proportion correct $=.96$ ) than among the English participants (mean proportion correct $=.83), t(9)=3.12, p<.02$, with adjusted degrees of freedom after Welch-Satterthwaite correction for larger variance in English than Himba participants.

\section{Discussion}

Our results are straightforward: The Ebbinghaus illusion is experienced by both English and Himba cultures but is experienced more strongly by English participants compared with Himba participants. In addition, both cultures showed a reduction in illusion when the inducing shape changed from similar (circle) to dissimilar (diamond). Before discussing these results, we first want to discount any explanations of the findings based on a misunderstanding of instructions by the Himba participants or cultural differences in familiarity with test procedures. In fact, by experiencing less illusion than the English participants, the Himba necessarily provided more accurate size judgments than the English participants. The two groups required equally little practice, showing that the task was easily understood by the Himba. It is possible that the amount of practice was insufficient to ensure that the Himba participants continued to make size judgments in the test trials. They could, for example, have been judging the distance between the two circles. Although we cannot rule this out completely, it provides an explanation similar to the one we give, namely attention to local detail. We also rule out that their responses were random choices; this would have provided no reliable difference across conditions. An alternative possibility of showing a bias toward selecting the larger inducers rather than the larger target would not have shown the observed effect of the size of the targets with large inducers. It also would not have produced a modulation of performance by inducer shape similar to that of the English participants. It is reasonable to maintain that the Himba participants understood the task and paid attention to it.

The second hypothesis under test was the possibility that the Himba participants would see more illusion than the English participants in a condition where inducers were dissimilar from the target. The linguistic difference between the English and the Himba could have implied that the former would be more likely than the latter to group basic shapes like circles and diamonds more readily into distinct categories (Roberson et al., 2002); it would have followed that the diamond inducers should have reduced the magnitude of the illusion to a greater extent in the English compared with the Himba participants. This was clearly not the case: The Himba participants reliably experienced less illusion overall and, like the English participants, showed a reduction in the illusion between circle and diamond inducers. These results, therefore, do not suggest that the Himba see circles and diamonds as similar. Thus, with respect to Roberson et al. (2002), an alternative to the impoverished naming explanation is now required for why the Himba categorized circles and squares together. It would seem more likely that a cognitive bias, such as local processing precedence, is responsible for the Himba forming many categories with small numbers of exemplars based on local detail.

Although there are many reports of visual illusions that reveal a difference between normal adult Western and isolated nonWestern observers (Segall, Campbell, \& Herskovitz, 1963, 1966), the current results are the first evidence of a visual illusion that demonstrates differences in the extent of grouping. The data seem to indicate that, when processing a configuration of multiple objects, the Himba do not show the precedence for global analysis that has been found in Western observers (Navon, 1977); instead, they are more likely to process the local features of a stimulus configuration. Thus, cultural variability in local versus global processing can lead to differences in subjective size perception, at least in the context of our task. A similar reduction in contextual sensitivity with the Ebbinghaus illusion has been shown in patients with autism (Dakin \& Frith, 2005), 4-year-old Western children (Kaldy \& Kovacs, 2003), and Western males (Phillips, Chapman, \& Berry, 2004). It is possible that these data too may be best explained by biases toward local processing.

\section{References}

Choplin, J. M., \& Medin, D. L. (1999). Similarity of the perimeters in the Ebbinghaus illusion. Perception \& Psychophysics, 61, 3-12.

Coren, S., \& Enns, J. T. (1993). Size contrast as a function of conceptual similarity between test and inducers. Perception \& Psychophysics, 54, 579-588.

Coren, S., \& Miller, J. (1974). Size contrast as a function of figural similarity. Perception \& Psychophysics, 16, 355-357.

Dakin, S., \& Frith, U. (2005). Vagaries of visual perception in autism. Neuron, 48, 497-507.

Davidoff, J. (1975). Differences in visual perception. London: Academic Press.

Deregowski, J. B. (1989). Real space and represented space: Cross-cultural perspectives. Behavioral and Brain Sciences, 12, 51-119.

Eckl, A. E. (2000). Language, culture and environment. The conceptualization of Herero cattle terms. In M. Bollig \& J.-B. Gewald (Eds.), History, cultural traditions and innovations in Southern Africa: Vol. 13. People, cattle and land. Transformation of a pastoral society in Southwestern Africa (pp. 401-431). Cologne, Germany: Ruediger Koeppe.

Evans-Pritchard, E. E. (1940). The Nuer. Oxford, England: Oxford University Press.

Happé, F. G. E. (1996). Studying weak central coherence at low levels: Children with autism do not succumb to visual illusions. A research note. Journal of Child Psychology and Psychiatry, 37, 873-877.

Happé, F. G. E., Briskman, J., \& Frith, U. (2001). Exploring the cognitive phenotype of autism: Weak 'central coherence' in parents and siblings of 
children with autism: I. Experimental tests. Journal of Child Psychology and Psychiatry, 42, 299-307.

Kaldy, Z., \& Kovacs, I. (2003). Visual context integration is not fully developed in 4-year-old children. Perception, 32, 657-666.

McNeil, J. E., \& Warrington, E. K. (1993). Prosopagnosia: A face-specific disorder. Quarterly Journal of Experimental Psychology: Human Experimental Psychology, 46(A), 1-10.

Navon, D. (1977). Forest before trees: The precedence of global features in visual perception. Cognitive Psychology, 9, 353-383.

Nisbett, R. E., \& Miyamoto, Y. (2005). The influence of culture: Holistic versus analytic perception. Trends in Cognitive Science, 9, 467-473.

Nisbett, R. E., Peng, K., Choi, I., \& Norenzayan, A. (2001). Culture and systems of thought: Holistic versus analytic cognition. Psychological Review, 108, 291-310.

Phillips, W. A., Chapman, K. L. S., \& Berry, P. D. (2004). Size perception is less context-sensitive in males. Perception, 33, 79-86.

Roberson, D., Davidoff, J., \& Shapiro, L. (2002). Squaring the circle: The cultural relativity of 'good' shape. Journal of Cognition and Culture, 2, $29-51$.
Roberson, D., Davies, I., \& Davidoff, J. (2000). Color categories are not universal: Replications and new evidence from a stone-age culture. Journal of Experimental Psychology: General, 129, 369-398.

Ropar, D., \& Mitchell, P. (1999). Are individuals with autism and Asperger's syndrome susceptible to visual illusions? Journal of Child Psychology and Psychiatry, 40, 1283-1293.

Rosch, E. H. (1973). Natural categories. Cognitive Psychology, 4, $328-350$.

Segall, M. H., Campbell, D. T., \& Herskovitz, M. J. (1963, February 22). Cultural differences in the perception of geometric illusions. Science, 139, 769-771.

Segall, M. H., Campbell, D. T., \& Herskovitz, M. J. (1966). The influence of culture on visual perception. Indianapolis, IN: BobbsMerrill.

Received February 7, 2006 Revision received October 23, 2006 Accepted October 30, 2006

\section{American Psychological Association SUBSCRIPTION ClaIMS INFORMATION}

Today's Date:

We provide this form to assist members, institutions, and nonmember individuals with any subscription problems. With the appropriate information we can begin a resolution. If you use the services of an agent, please do NOT duplicate claims through them and directly to us. PLEASE PRINT CLEARLY AND IN INK IF POSSIBLE.

\begin{tabular}{l}
\hline PRINT FULL NAME OR KEY NAME OF NNSTITUTION \\
\hline ADDRESS \\
\hline CITY
\end{tabular}

YOUR NAME AND PHONE NUMBER

TITLE
MEMBER OR CUSTOMER NUMBER (MAY BE FOUND ON ANY PAST ISSUE LABEL) DATE YOUR ORDER WAS MAILED (OR PHONED)

\section{PREPAID CHECK CHARGE}

CHECK/CARD CLEARED DATE:

(If possible, send a copy, front and back, of your cancelled check to help us in our research of your claina.)

ISSUES: __ MISSLNG _ DAMAGED

Thank you. Once a claim is received and resolved, delivery of replacement issues routinely takes 4-6 weeks.

(TO BE FILLED OUT BY APA STAFF)

DATE RECEIVED:

ACTION TAKEN:

STAFF NAME:
DATE OF ACTION:

INV. NO. \& DATE:

LABEL NO. \& DATE:

Send this form to APA Subscription Claims, 750 First Street, NE, Washington, DC 20002-4242

PLEASE DO NOT REMOVE. A PHOTOCOPY MAY BE USED. 